\title{
Temporary Tattoos with Lasting Consequences
}

Jonathan Cubitt ${ }^{1^{*}}$, Marc C Swan ${ }^{2}$ and Michael P Tyler ${ }^{2}$

${ }^{1}$ The Welsh Centre for Burns and Plastic Surgery Morriston Hospital, Morriston, Swansea, UK

${ }^{2}$ Regional Burns Unit Stoke Mandeville Hospital Mandeville Road Aylesbury Buckinghamshire, UK

${ }^{*}$ Corresponding Author: Jonathan Cubitt, The Welsh Centre for Burns and Plastic Surgery, Morriston Hospital, Morriston,Swansea, SA6 6N; Email: jonathan.cubitt@wales.nhs.uk

Received date: February 05, 2015, Accepted date: March 11, 2015, Published date: March 21, 2015

Copyright: () 2015 Cubitt J, et al. This is an open-access article distributed under the terms of the Creative Commons Attribution License, which permits unrestricted use, distribution, and reproduction in any medium, provided the original author and source are credited.

\begin{abstract}
:
Body art and tattoos have become a ubiquitous part of modern culture and the popularity and availability of unregulated temporary henna-based tattoos has increased. The addition of para-phenylenediamine to natural henna makes the tattoo set quicker and gives a darker coloration; however, it is a potent allergen. We describe the case of an 11 year old girl who developed a Type IV delayed-type hypersensitivity reaction to a black henna tattoo. This case highlights the importance of public education about potent allergens that may be encountered in products such as henna tattoos.
\end{abstract}

Keywords: Henna; Tattoo; Delayed type IV allergic reaction; Paraphenylenediamine

\section{Introduction}

Body adornment by tattooing has been used for thousands of years in human culture. Early tribal and warrior origins have given way to their widespread use in popular culture with musicians, actors and athletes being decorated with tattoos. Permanent tattoos come at a not insignificant financial cost, but also, in the context of less fastidious tattoo artisans, have the potential risk of transmission of blood borne infections. Furthermore their permanence might outlive the bearer's affinity with their chosen motif; complete scarless removal of tattoo pigment is rarely possible [1]. For these reasons, and the greater propensity for foreign travel, temporary tattoos are becoming increasingly popular in modern Western society.

Henna has been used for centuries in many cultures as a dying agent for hair and body painting. When applied directly to the surface of the skin it stains the dermis a reddish-brown hue (mediated by the agent 2-hydroxy-1, 4-naphthoquinolone) which lasts for several weeks. Pure henna is a plant extract from Lawsonia inermis, a member of the Lythraceae family. Adverse reactions to henna are extremely rare, although life-threatening haemolysis has been reported in individuals with glucose-6-phospate dehydrogenase deficiency $[2,3]$. The recent introduction of 'black henna', which contains the synthetic aniline derivative Para-Phenylenediamine (PPD), has created a significant public health issue due to the risk of hypersensitivity reactions. No natural black henna exists. Approximately $6 \%$ of dermatitis patients have a positive patch test reaction to $\mathrm{PPD}$, indicating previous contact sensitisation, which, in approximately $40 \%$ of patients is related to the use of hair dye [4].

Black henna is becoming increasingly popular as it accelerates the dying process, amplifies the intensity of the tattoo mark (being a dark brown-black colour) and prolongs its longevity [5]. Temporary tattooing may cause significant and lifelong sensitisation, which can have implications for numerous patients who will subsequently become exposed to hair dyes, rubber chemicals and textile dyes. PPD is a potent T-cell activator and, in 2006, was awarded the accolade of 'Allergen of the Year' by the American Contact Dermatitis Society [6]. The US Food and Drug Administration restrict its use to hair dyes; it is not approved for topical cutaneous applications. Black henna pastes may contain up to ten times the concentration of PPD that is recommended for use in proprietary hair-colouring products [2]. Many European countries, including France and Germany, have banned the use of PPD in hair dyes.

\section{Case Report}

An eleven year-old healthy Caucasian girl had a temporary henna tattoo applied by a local street artist while on holiday in the Sinai Peninsula, Egypt. The black henna mixture was applied to the skin on the volar aspect of her left forearm in the form of Arabic script and on her left lower leg in the profile of a dolphin. The henna was applied as a black paste and left to dry and fall off naturally. No injectable ink used. She had no previous exposure to henna or hair dyes and had no history of allergy or atopy. Her older sibling also had a henna tattoo conducted by the same artisan, but he failed to exhibit any complication.

Ten days following the tattoo she developed swelling and erythema and a marked papulovesicular dermatitis localised to the precise contours of the henna tattoo. Having returned to the UK, her general practitioner referred her to the Regional Burns Unit where she was reviewed 12 days following initial exposure. Her wounds (Figure 1) were minimally de-roofed and managed as a superficial partial thickness burns with a silicone based dressing (Adaptic, Johnson \& Johnson). The leg was cellulitic on presentation although she exhibited no symptoms or signs of a systemic inflammatory response (temperature $37.2^{\circ} \mathrm{C}$ ) nor was there any associated lymphadenopathy. She was commenced on an empirical course of oral Flucloxacillin; wound cultures subsequently demonstrated no significant growth. Topical corticosteroids were not started at this juncture, but antihistamines were advised to provide symptomatic relief of her pruritis.

On review three days later, the leg cellulitis had completely resolved and the wounds had largely epithelialised (Figure 2). Four weeks after 
exposure, the wounds had completely healed but exhibited widespread hypopigmentation (Figure 3). The patient was advised to moisturise the affected regions on a daily basis and to protect them from excessive sun exposure. Skin patch testing was not performed. She was warned of the potential risk of future exposure to PPD containing hair dyes.

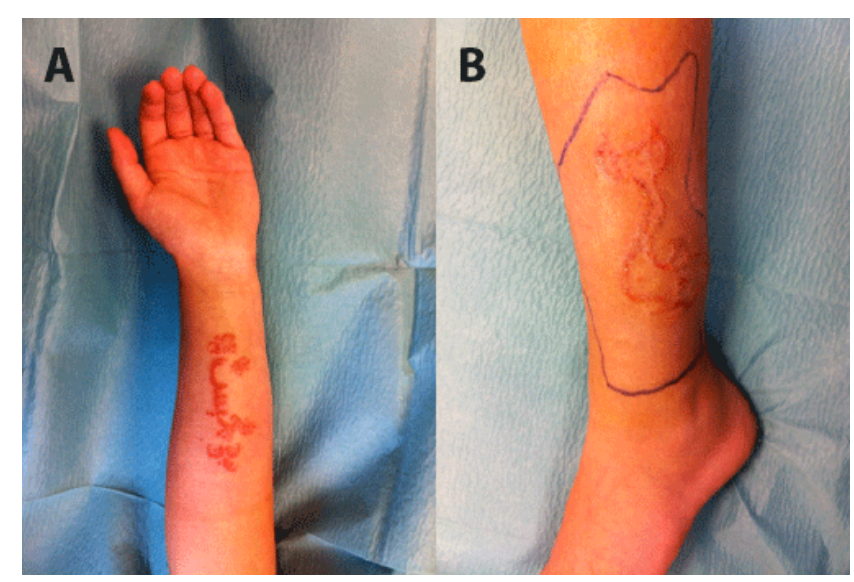

Figure 1: Appearance of patient's left volar forearm (a) and left lateral leg (b) 12 days after application of the black henna tattoo. The area of cellulitis in the lower leg is delineated by the blue skin marker.

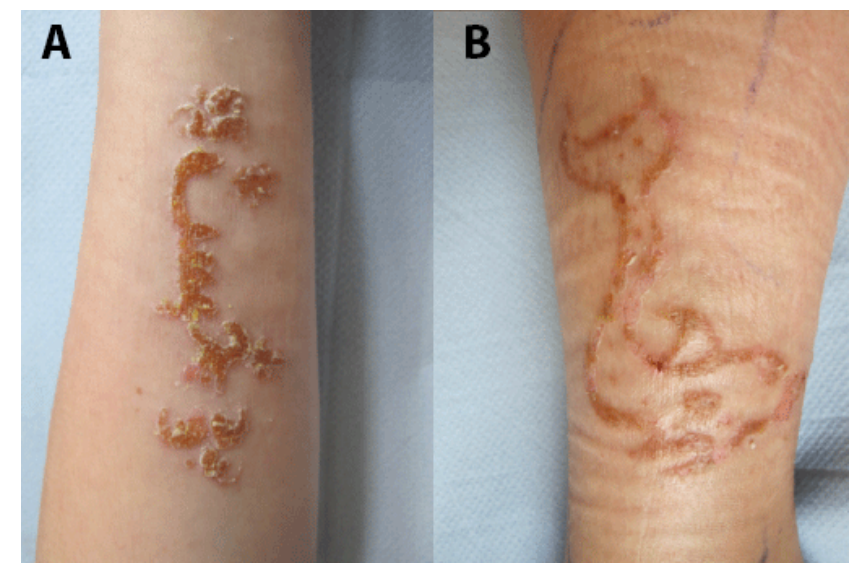

Figure 2: Appearance of patient's left volar forearm (a) and left lateral leg (b) 15 days after application of the black henna tattoo.

\section{Discussion}

The patient displayed a Type IV delayed-type hypersensitivity reaction to the black henna mixture with sensitisation occurring during prolonged contact to the dye rather than from previous PPD exposure (in which case an intense dermatitis reaction would have occurred within 48 hours of the re-exposure). The potency of PPD as an allergen is directly related to its concentration and the duration of exposure to the skin; as vendors of black henna are entirely unregulated, the concentration of PPD can reach up to $16 \%$, far in excess of that permissible in hair dyes $[2,7,8]$. Discordance between siblings who are both exposed to the same allergen is well recognised in the context of henna tattoos, with certain genotypes being more susceptible to contact allergy to PPD [9].

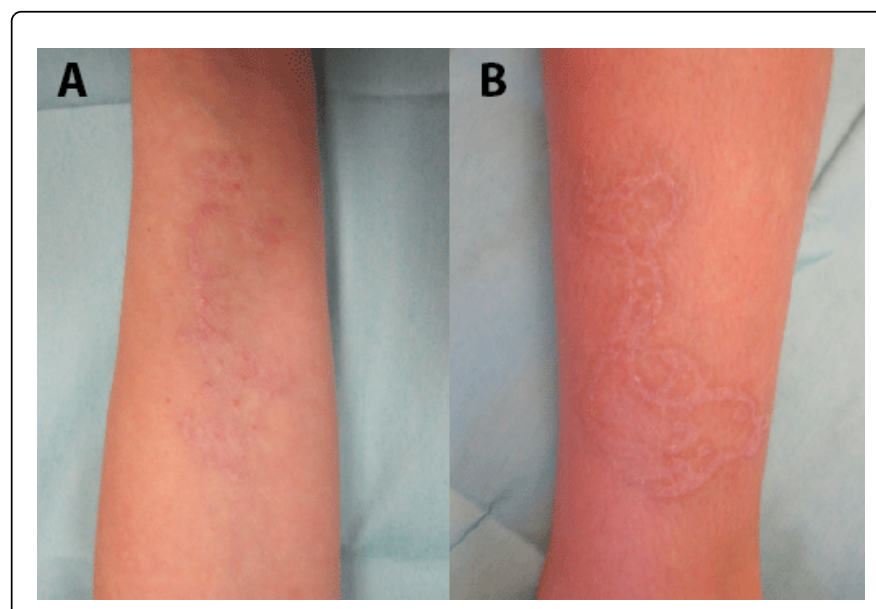

Figure 3: Appearance of patient's left volar forearm (a) and left lateral leg (b) four weeks after application of the black henna tattoo.

Numerous similar cases of allergic contact dermatitis to PPD have been reported in the literature $[2,3,7,9]$. The spectrum of the adverse consequences ranges from mild eczema to more severe bullous reactions with permanent scarring, and in some instances, keloid scar formation. Rarely, acute life threatening Type I immediate hypersensitivity reactions have also been described [10].

Aims to regulate those who pedal black henna tattoos in beach resorts and market squares across the globe are unlikely to be wholly successful due to the peripatetic nature of the trade; indeed many patients are unaware of the complications of PPD exposure until they have returned to their country of origin. Perhaps public health strategy should focus on educating travellers of the potential risks of temporary tattoos, particularly in the population who have had a previous adverse reaction to henna or hair dye. Nonetheless, the application of unadulterated red-brown henna is likely to be safe, except in those individuals with glucose-6-phospate dehydrogenase deficiency $[3,8]$.

The long-term sequelae of PPD exposure include scarring and postinflammatory dyschromia of the tattoo site, as well as a lifelong hypersensitivity to PPD, which may have occupational as well as lifestyle implications. Thus the notion that black henna represents a 'temporary' cosmetic embellishment is unfortunately all too frequently far from the truth.

\section{References}

1. Kent KM, Graber EM (2012) Laser Tattoo Removal: A Review. Dermatologic Surgery 38: 1-13.

2. Brancaccio RR, Brown LH, Chang YT, Fogelman JP, Mafong EA, et al. (2002) Identification and quantification of para-phenylenediamine in a temporary black henna tattoo. Am J Contact Dermat 13: 15-18.

3. Urkin J (2006) Henna tattooing dermatitis: consider an additive as the culprit. Br J Gen Pract 56: 794-795.

4. Thyssen JP, White JM (2008) Epidemiological data on consumer allergy to p-phenylenediamine. Contact Dermatitis 59: 327-343.

5. Jacob SE, Brod BA (2011) Paraphenylenediamine in black henna tattoos: sensitization of toddlers indicates a clear need for legislative action. J Clin Aesthet Dermatol 4: 46-47.

6. DeLeo VA (2006) p-Phenylenediamine. Dermatitis 17: 53-55. 
Citation: Jonathan Cubitt, Marc C Swan and Michael P Tyler (2015) Temporary Tattoos with Lasting Consequences. J Allergy Ther 6: 206. doi: 10.4172/2155-6121.1000206

Page 3 of 3

7. Sonnen G (2007) Type IV hypersensitivity reaction to a temporary tattoo. Proc (Bayl Univ Med Cent) 20: 36-38.

8. Almeida PJ, BorregoL, Pulido-Melian E, et al.(2012) Quantification of $\mathrm{p}$ phenylenediamine and 2-hydroxy-1,4-naphthoquinone in henna tattoos. Contact Dermatitis 66: 33-37.
9. Hardwicke J, Azad S (2006) Temporary henna tattooing in siblings--an unusual chemical burn. Burns 32: 1064-1065.

10. Onder M, Atahan CC, Oztas P, et al. (2001) Temporary henna tattoo reactions in children. Int J Dermatol 40: 577-579. 